\title{
Validity of self-reported diabetes varies with sociodemographic charecteristics: Example from Iran
}

\author{
Mehdi Moradinazar ${ }^{\mathrm{a}}$, Yahya Pasdar ${ }^{\mathrm{b}}$, Farid Najafi ${ }^{\mathrm{a}}$, Ebrahim Shakiba ${ }^{\mathrm{c}}$, Behrooz $\operatorname{Hamzeh}^{\mathrm{d}, *}$, \\ Mehnoosh Samadi $^{\mathrm{b}}$, Maryam Mirzaei ${ }^{\mathrm{a}}$, Annette J. Dobson ${ }^{\mathrm{e}}$ \\ ${ }^{a}$ Research Center for Environmental Determinants of Health School of Public Health, Kermanshah University of Medical Sciences, Kermanshah, Iran \\ ${ }^{\mathrm{b}}$ Nutritional Sciences Department School of Public Health, Kermanshah University of Medical Sciences, Kermanshah, Iran \\ ${ }^{\mathrm{c}}$ Medical School, Kermanshah University of Medical Sciences, Kermanshah, Iran \\ ${ }^{\mathrm{d}}$ Department of Public Health, Kermanshah University of Medical Sciences, Kermanshah, Iran \\ ${ }^{\mathrm{e}}$ School of Public Health, University of Queensland, Herston, Queensland, Australia
}

A R T I C L E IN F O

\section{Keywords:}

Diabetes

Cohort study

Validity

Self-report

\begin{abstract}
A B S T R A C T
Introduction: The purpose of this study was to examine the validity of self-report diabetes according to sociodemographic characteristics in participants of Ravansar Non-Communicable Diseases (RaNCD) cohort study. Methods: The study population included 10182 adults (35-65 years old) participating in RaNCD. The 'gold standard' for diagnosis of diabetes mellitus was taken as a history of relevant medicine use and/or measurement of fasting blood glucose indicating diabetes. Self-report data collected before the clinical examinations through interviews by well-trained staff. Sensitivity, specificity, and positive and negative predictive values were used to estimate the accuracy of self-report in diabetes.

Results: Of the 10182 individuals invited to participate in the RaNCD, 96 people were not willing to participate in the study (participation rate $=99.06 \%$ ). The prevalence of diabetes by self-report and gold standard was $10.04 \%$ and $8.19 \%$ respectively. Among those who said they had diabetes, 389 (38.78\%) did not have diabetes. Self-reported diabetes had sensitivity, specificity, positive and negative predictive values of $75.0 \%, 95.8 \%$, $61.2 \%$ and $97.7 \%$, respectively. Being female, aging, smoking, and having a family history of diabetes increased the chance of false positive; and being male, having a family history of diabetes, aging, and increasing BMI increased the chance of false negative.

Conclusions: Although the overall validity of self-report in diabetes seems to be acceptable in the study population with a correction factor of $25.3 \%$, it should be noted that the is influenced by age, gender, and family history of diabetes.
\end{abstract}

\section{Introduction}

Diabetes mellitus (DM), which is known as a metabolic disorder, has been recognized as a latent epidemic in recent years. ${ }^{1,2}$ According to the International Diabetes Federation, 2015 witnessed 415 million diabetics in the world, with an increasing trend. ${ }^{1}$ According to previous studies, diabetes accounts for 1.3 million deaths in the world and about 56 million total Disability-Adjusted Life Years (DALY). Among WHO's six regional offices around the world, Eastern Mediterranean is known to be the one with the highest prevalence of diabetes. The prevalence of diabetes in Iran is estimated to be 6.1 to $9.8 \%{ }^{1-5}$ Considering the growing trend of this disease in the world and its high economic burden on societies, its detection is very important as one of the most important health issues in many societies. ${ }^{2,4}$

There are different methods for assessment of prevalence and incidence of diabetes in populations. While medication measurement plus checking blood sugar is a standard method, use of self-report as an easy method, is used frequently to diagnose diabetes and determine its prevalence. ${ }^{6}$ Previous studies have shown that gold standards for the detection of a disease are sometimes difficult, and due to their cost and lack of easy access to all required documents are often not used in large population-based studies. Therefore, self-reporting is sometimes used in population-based epidemiological studies because of its low cost and simplicity to collect information along with gold standards such as

\footnotetext{
* Corresponding author. School of Health, Kermanshah University of Medical Sciences, Kermanshah, Iran.

E-mail addresses: m.moradinazar@kums.ac.ir (M. Moradinazar), yahya.pasdar@gmail.com (Y. Pasdar), farid_n32@yahoo.com (F. Najafi), e.shakiba@yahoo.com (E. Shakiba), behrooz_hamzeh@yahoo.com (B. Hamzeh), mehnoosh_samadi@yahoo.com (M. Samadi), mirzaei.m.epid92@gmail.com (M. Mirzaei), a.dobson@sph.uq.edu.au (A.J. Dobson).
} 
medication records and clinical measurements. ${ }^{7-10}$

However, detecting the prevalence of diabetes may not be accurate through self-reporting and the validity of self-reporting may vary in different groups and it might be influenced by the recall bias and wish bias of the participants. Therefore, the accuracy and validity of selfreporting are usually assessed in studies around the world. ${ }^{7,9}$

A review of studies conducted on the agreement between self-report on diabetes incidence and medication records shows different results. Comparing the results of various studies, we should notice the difference in methodology and demographics of the subjects in different studies. $^{9,10}$ Although population-based studies have confirmed the validity of self-report diabetes, they still emphasize that the self-report method is influenced by factors such as social rejection and the underlying diseases in different ethnic groups. Therefore, self-report studies should be cautiously analyzed, and the validity of self-report need to be measured. ${ }^{11,12}$

The objective of validation studies is to measure the level of agreement and the accuracy of self-reporting data with gold standards including diagnosis obtained through history of relevant medicine use (insulin and/or oral hypoglycemic agents) and measurements of blood markers (fasting blood glucose). ${ }^{7,9}$ Considering the contradictions in the results of previous studies and the lack of similar studies, this study aimed to investigate factors associated with the validity of self-report in prevalent cases of diabetes in Ravansar Non-Communicable Diseases (RaNCD) cohort study.

\section{Method}

\subsection{Study population}

The RaNCD cohort study is part of the PERSIAN (Prospective Epidemiological Research Studies in IrAN) Cohort, focused on permanent residents of Ravansar aged 35-65 years. In the PERSIAN cohort, all 18 cohort sites used the same questionnaire containing different sections: general information (demographic, socioeconomic status, life style factors, occupational history, mobile use and physical activity), medical section (past medical history, past and present medicine use, family medical history, obstetric and gynecology history, oral health and personal habit) and nutrition questionnaire (food frequency questionnaire, dietary habit, water use and supplement). The details of the study design and rationale for conducting the PERSIAN cohort have been presented elsewhere. ${ }^{13,14,15}$

\subsection{Data collection and measurements}

In order to collect information, participants were invited to the study site. After registration of participants, the anthropometric measures assessed by bioelectric impedance. To measure weight, we used a Bio Impedance Analyzer BIA (InBody 770 BIOSPACE, KOREA). Height was measured with $0.1 \mathrm{~cm}$ accuracy with stadiometer. Body mass index (BMI) was calculated as weight in kilograms divided by height in meters squared. Age was determined based on the age of the person's birth certificate and the actual age was not standard. The criteria for determining the level of education were the last degree. Blood pressure was measured using Aneroid Blood Pressure Monitor after 15 min of rest with two measurements of right arm and two measurement of left arm. Average of four measurements considered as mean of systolic and diastolic blood pressure. This study considered people with a systolic blood pressure of 139 or higher and/or a diastolic blood pressure of 89 or higher and/or those with a history of using antihypertensive drugs as hypertensive. Smoking status evaluated by asking about smoking of at least 100 cigarettes throughout the life. ${ }^{16}$

Family history was studied in first and second degree relatives A second-degree relative (SDR) is someone who shares $25 \%$ of a person's genes. It includes uncles, aunts, nephews, nieces, grandparents, grandchildren, half-siblings, and double cousins.
For the purpose of this study gold standard for diagnosis of diabetes mellitus defined as positive history of relevant medicine use (insulin and/or oral hypoglycemic agents) and/or a fasting blood glucose indicating diabetes. Fasting blood sugar $(\mathrm{FBS})>=126 \mathrm{mg}$ per $\mathrm{dL}$ [7 mmol per L] defined as diabetes mellitus. we used wealth index as proxy for economic status. ${ }^{16}$ Wealth index was generated using principal component analysis (PCA) on data of durable goods, housing characteristics and other amenities.

\subsection{Statistical analysis and calculations}

Validity was assessed using sensitivity, (true positives/[true positives plus false negatives]), specificity (true negatives/[true negatives plus false positives]), positive predictive value (true positives/[true positives plus false positives]), negative predictive value (true negatives/[true negatives plus false negatives]). Positive likelihood ratio (LR +) (sensitivity/[1-specificity]), and negative likelihood ratio (LR-) ([1sensitivity]/specificity) was calculated. For all these values, $95 \%$ confidence intervals calculated using standard methods for proportions.

Univariable and multivariable logistic regression were used to examine the discordance between self-report and gold standard. A twosided alpha level of 0.05 was considered statistically significant. All analyses were carried out using Stata software (version 14.1) (Stata Corp, College Station, TX, USA).

\subsection{Ethical statement}

The present study was conducted according to the Helsinki Declaration. The study was approved by the ethics committee of the vice chancellery of research and technology, Kermanshah University of Medical Sciences (KUMS.REC.1394.315) and the written informed consent was obtained from each participant after explaining the purpose of research.

\section{Results}

Of the 10182 individuals invited to participate in the RaNCD, 96 people were not willing to participate in the study (participation rate $=99.06 \%$ ) and there were no self-reporting and measuring FBS for 85 individuals. Of the 10001 remaining participants, 4742 (47.42\%) were male and $5259(52.58 \%)$ were female. The mean age of the male participants was $46.99 \pm 8.2$ and the mean age of the female participants was $47.49 \pm 8.4$ years. As Table 1 shows, 4607 (46.07\%) of the participants in the RaNCD were illiterate and 9090 (90.19\%) were married (Table 1). The prevalence of self-report diabetes and diabetes measured by gold standard method was 1003(10.04\%) and 819(8.19\%) respectively.

From total of those with high FBS and/or those who received treatment, 205 (25.03\%) did not know about their diabetes, respectively. From total of 819 participants with confirmed diabetes 465(56.77\%) cases were under treatment. Among people taking medicine, $310(37.85 \%)$ did not adequately control their diabetes (they had FBS $>=126 \mathrm{mg} / \mathrm{dl}$ ). Among those who said they had diabetes (1003 people), 389 (38.78\%) did not have confirmed diabetes(Fig. 1).

The accuracy of self-reported diabetes was $94.05 \%$. Overall, selfreported diabetes had sensitivity, specificity, positive and negative predictive values of $75.0 \%, 95.8 \%, 61.2 \%$, and $97.7 \%$, respectively. The agreement rate based on the Kappa test was $64.0 \%$ (Table 2).

Investigation of the factors affecting discordance at the multivariable level suggested that increasing age, higher BMI and having family history of diabetes plus smoking increased the overall discordance. However higher education decrease the likelihood of total discordance. Smokers, females, older people and those who had family history of diabetes were more likely to report diabetes without confirmation by gold standard (false positives). While for false-negative report of diabetes, family history of diabetes, older age and higher BMI 
Table 1

Demographic characteristic of participant in RaNCD by sex.

\begin{tabular}{|c|c|c|c|c|c|}
\hline Variables & & Total N(\%) & Male N(\%) & Female N(\%) & $\mathrm{P}$ value \\
\hline \multirow[t]{6}{*}{ Age group (years) $(\mathrm{N}=10001)$} & $35-40$ & $2639(26.39)$ & $1274(48.28)$ & 1365(51.72) & $<0.001$ \\
\hline & $41-45$ & $2098(20.98)$ & 998(47.57) & $1100(52.43)$ & \\
\hline & $46-50$ & 1763(17.63) & $875(49.63)$ & $888(50.37)$ & \\
\hline & $51-55$ & $1432(14.32)$ & 693(48.39) & $739(51.61)$ & \\
\hline & $56-60$ & $1311(13.11)$ & $613(46.76)$ & $698(53.24)$ & \\
\hline & $60-65$ & $758(7.58)$ & $289(38.13)$ & $469(61.87)$ & \\
\hline \multirow[t]{2}{*}{ Marital status $(\mathrm{N}=10001)$} & Single & $981(9.81)$ & $135(13.76)$ & $846(86.24)$ & $<0.001$ \\
\hline & Married & $9090(90.19)$ & $4607(51.08)$ & $4413(48.92)$ & \\
\hline \multirow[t]{5}{*}{ Length of education (year) $(\mathrm{N}=10001)$} & illiterate & $4607(46.07)$ & $1302(28.26)$ & $3305(71.74)$ & $<0.001$ \\
\hline & Primary & $2610(26.10)$ & $1327(50.84)$ & $1283(49.16)$ & \\
\hline & Lower secondary & 1059(10.59) & 745(70.35) & $314(29.65)$ & \\
\hline & Higher secondary & $966(9.66)$ & $752(77.85)$ & $214(22.15)$ & \\
\hline & Academic & $759(7.59)$ & $616(81.16)$ & $143(18.84)$ & \\
\hline \multirow[t]{2}{*}{ Place $(\mathrm{N}=10001)$} & Rural & 4091(40.91) & $1837(44.90)$ & $2254(50.10)$ & $<0.001$ \\
\hline & Urban & $5910(59.09)$ & $2905(49.15)$ & $3005(50.85)$ & \\
\hline
\end{tabular}

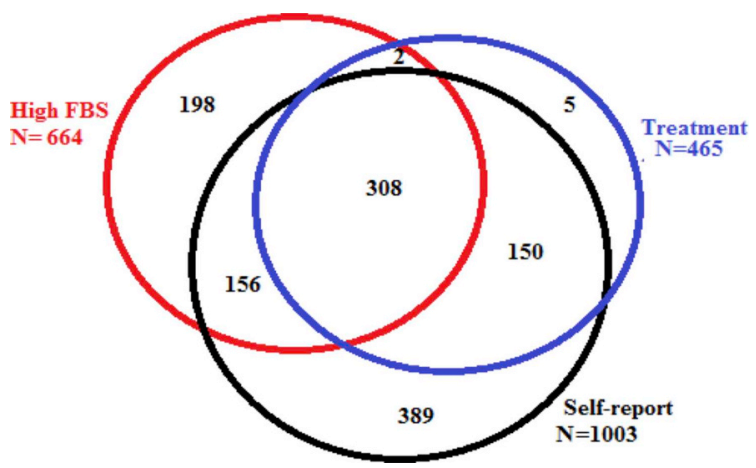

Fig. 1. Frequency of self-report diabetes and diabetes measured by gold standard(treatment -high FBS).

increased the likelihood, women were both less likely wrongly report they did not have diabetes and high likely to wrongly report they did have diabetes (Table 3).

\section{Discussion}

In population-based epidemiological studies, either self-report or clinical gold standards, is a common tool for assessing the prevalence and incidence of chronic diseases. ${ }^{7}$ The main objective of this study was to assess the effect of sociodemographic factors on validity of self-report diabetes using data from a large population based data in Iran. In the present study, although, the participants' self-report on diabetes had a sensitivity of $75.0 \%$, specificity of $95.8 \%$, positive predictive value of $60 \%$ and negative predictive value of $97.7 \%$, such values were largely dependent to sociodemographic variables. In our study, near $43 \%$ of all participants with diabetes received no treatment (354 from total of 819). Such findings are in line with previous reports and provide an update figure from validation of self-report of diabetes in Iran. ${ }^{17}$

The results revealed that although the overall agreement between the participants' self-report responses and gold standards was acceptable, it largely depends on sociodemographic variables. Our results are consistent with the results of similar studies reporting that self-report in diabetes is more accurate than self-report in other chronic diseases. ${ }^{18,19}$ This finding can be justified in that the accuracy of self-report is more likely to be confirmed in diseases with clearer and more specific diagnostic criteria (such as diabetes) and those with acute onset. ${ }^{718}$ However, all such studies reported that self-report is highly influnenced by sociodemographic factors $(7,16,17)$

For example, the results of a population-based study by Goldman et al. to validate self-report in diabetes and hypertension in 2003 also showed that self-report provide an acceptable estimate of the prevalence of diabetes, but varies with sociodemographic factors such as age and education. ${ }^{8}$

Moderate sensitivity (75.0\%) versus high specificity (95.8\%) of selfreport in this study was in line with the results of similar studies of validation of self-report in diabetes. ${ }^{2,20}$ While older results from Iran reported a lower sensitivity (61.5\%) in 2003 and 2004 (2), someone predict to have a higher sensitivity for those recent roports. Higher awareness and better care provided by health care system are two important factors affecting such increase in accuracy of self-report diabetes. This moderate sensitivity versus high specificity and underestimation of diabetes prevalence can be attributed to the characteristics of study population (age structure, sex, education and SES), as well as the criteria for the definition of gold standard for diabetes, which might be different in each study. ${ }^{18}$

The agreement rate between self-report in diabetes and the gold standards was moderate (Kappa coefficient $=0.64$ ), which is in line with the results of similar studies. Diabetes is one of the chronic diseases with a moderate Kappa coefficient of agreement for self-report in similar studies ranging from 0.6 to $0.9 .^{8,21,22}$ For example, a population-based study in Canada and Spain on self-report in diabetes reported a Kappa coefficient of agreement 0.81 and 0.78 , respectively ( 9 and 20). Overall, in similar population-based studies, self-report in diabetes has a moderate sensitivity, high specificity and a moderate Kappa.

In the multivariable analysis, demographic variables including age, sex, smoking, BMI, and family history of diabetes were related to discordance after adjusting other factors related to self-report. Similar to the results from elsewhere, our results revealed false positive and false negative rates increased by age (9). This may reflect a recall bias (due to

Table 2

Sensitivity and specificity of self-reported of DM in RaNCD.

\begin{tabular}{|c|c|c|c|c|c|c|c|c|c|c|c|}
\hline & & \multicolumn{2}{|c|}{ Gold standard } & \multirow{2}{*}{$\begin{array}{l}\text { Accuracy } \\
-(95 \% \mathrm{CI})\end{array}$} & \multirow{2}{*}{$\begin{array}{l}\text { Kappa } \\
(95 \% \mathrm{CI})\end{array}$} & \multirow{2}{*}{$\begin{array}{l}\text { Sensitivity } \\
(95 \% \mathrm{CI})\end{array}$} & \multirow{2}{*}{$\begin{array}{l}\text { Specificity } \\
(95 \% \mathrm{CI})\end{array}$} & \multirow[t]{2}{*}{$\mathrm{LR}+(95 \% \mathrm{CI})$} & \multirow[t]{2}{*}{ LR- $(95 \% \mathrm{CI})$} & \multirow{2}{*}{$\begin{array}{l}\text { PPV } \\
(95 \% C I)\end{array}$} & \multirow{2}{*}{$\begin{array}{l}\text { NPV } \\
(95 \% C I)\end{array}$} \\
\hline & & No & Yes & & & & & & & & \\
\hline Self-reported DM & $\begin{array}{l}\text { No } \\
\text { Yes }\end{array}$ & $\begin{array}{l}8793 \\
389\end{array}$ & $\begin{array}{l}205 \\
614\end{array}$ & $\begin{array}{l}94.05 \text { (93.6- } \\
94.5)\end{array}$ & $\begin{array}{l}64.0(61.5- \\
66.6)\end{array}$ & $\begin{array}{l}75.0 \text { (71.9- } \\
77.9)\end{array}$ & $\begin{array}{l}95.8 \text { (95.3- } \\
96.2)\end{array}$ & $\begin{array}{l}17.67(15.91- \\
19.63)\end{array}$ & $\begin{array}{l}0.26(0.23- \\
0.29)\end{array}$ & $\begin{array}{l}61.2(58.1- \\
64.2)\end{array}$ & $\begin{array}{l}97.7 \text { (97.4- } \\
98.0)\end{array}$ \\
\hline
\end{tabular}




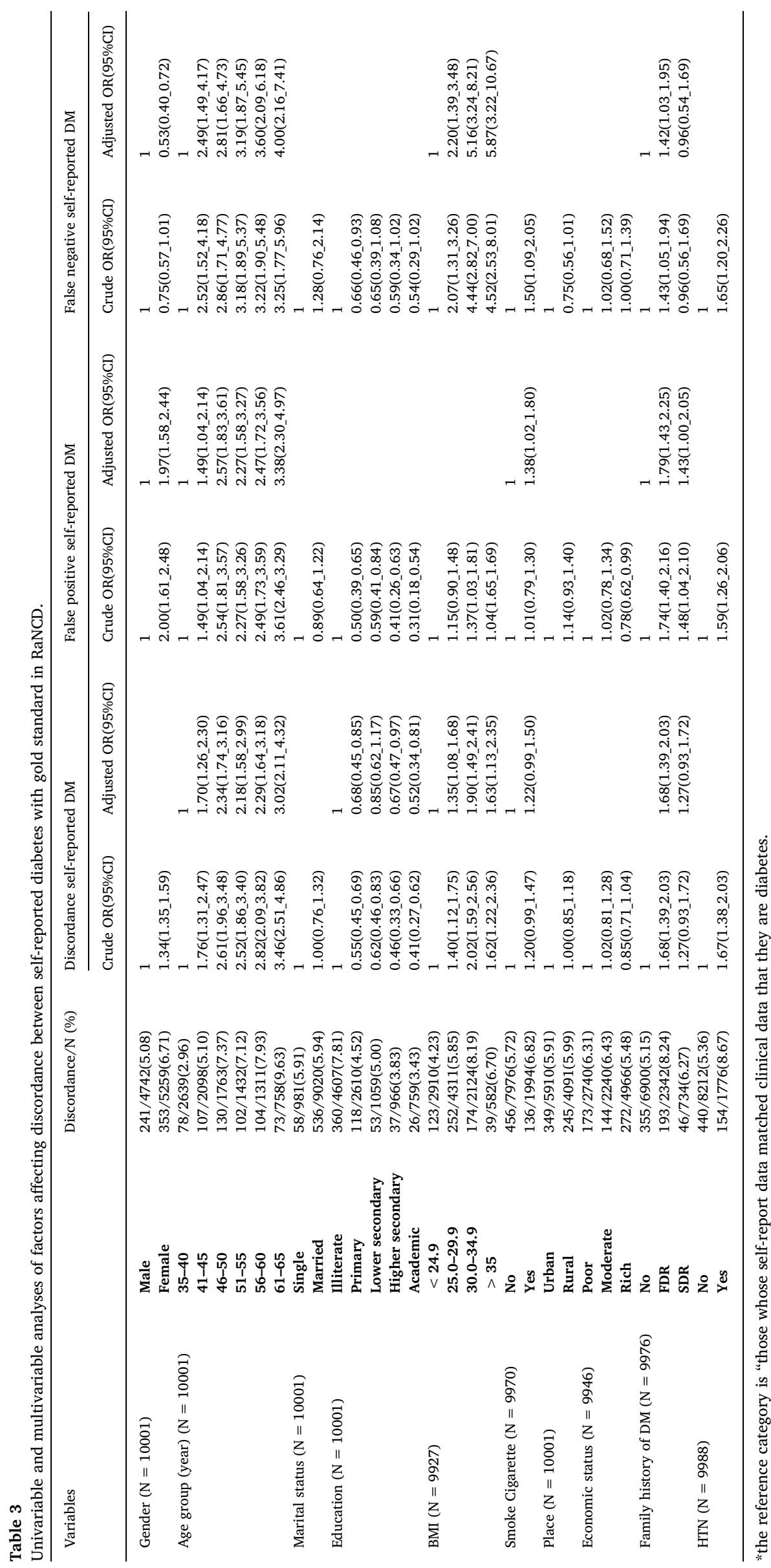


depression and other age-related disabilities) or actual unawareness about diabetes due to the failure to undergo blood glucose testing. ${ }^{7,8,23}$

In our study, self-report of diabetes among women were more likely to have a discordance with gold standard. They had also more false positives and less false negatives compared with men, which can be attributed to better self-care and more and timely use of health services among women. ${ }^{7,8,23}$ In line with these findings, studies from elsewhere reported women had better dietary self-care behaviours about diabetes, for example they were more likely than men to count carbohydrates and reduce their consumption of dietary fat. ${ }^{22}$ Thus women may pay more scrupulous attention to diabetes. At the level of multivariable analysis and by adjusting other factors affecting discordance, the increase in BMI increased the likelihood of false negative rate, but no relationship was observed between BMI and false positive rate of selfreported diabetes. Huerta et al. (2009) reported that likelihood of positive self-reports of diabetes increased with obesity. ${ }^{9}$ In fact, obesity is probably the most important factor in the development of insulin resistance. People who are overweight or obese have added pressure on their body's ability to use insulin to properly control blood sugar levels therefore they are more likely to develop diabetes. In addition, overweight and obesity are associated with poor health and such people may also be careless about obesity related risks.

The relationship between the positive family history of diabetes and the level of discordance was significant, which was correct for both false positive and negative self-reported diabetes. our findings is similar to study in Spain in which subjects with a family history of diabetes reported more frequently that they had diabetes. ${ }^{24}$ People with a positive family history of diabetes are assumed to be more aware of the diabetes and therefore they can report false positive report of diabetes. However, increase in likelihood of false negative report of diabetes is really difficult to be justified.

Our findings revealed that smokers are more likely to falsely report diabetes without any effect on false negative report. Smoking is now proven to be an independent risk factor for diabetes, and amongst diabetics it increases the risk of complications. ${ }^{25}$ Different reports demonstrated that most smokers know that smoking is a risk factor for their general health and in fact individuals with good to excellent selfrated health are those who have never smoked. We can explain that smokers worry about their health and harm of smoking that have a false positive report about their blood glucose status.

In present study, although, level of education was associated with lower likelihood of total discordance between self-reported diabetes and diabetes diagnosed by gold standard, there is no association between false positive andnegative (separately) with gold standard in multivariable analysis.

In line with the results of this study, there was a significant relationship between self-report accuracy and demographic variables such as age, educational level, positive family history, gender and BMI in studies conducted in other countries. ${ }^{7,8,26,27}$

The association between demographic characteristics and validity of self-reported diabetes indicates that such specific characteristics affect the patient's awareness, and should be considered in interpreting the results.

\subsection{Weaknesses and strengths}

Certain specific limitations should be taken into account when interpreting the results. Validity results may depend on the specific nature of the study; it can be a function of the demographic characteristics as well as socio-cultural background in each population. We have reported the validity of self-reported prevalent cases of diabetes. We assume that our finding might be different from those who have studied the incident cases. In fact, the design and methodology of the studies are different. Therefore, these issues should be considered when generalizing the results. However, diabetes is well-known chronic disease with clear criteria for diagnosis and therefore there is no variability for definition of such condition over different studies.

The present study is the largest and the first study in Kurdish region of Iran. RaNCD is part of PERSIAN cohort designed by a central team with cooperation of 18 other centers all from Iranian universities. In Iran, there is a very well-known health system providing primary health care for the whole population from large cities to remote areas. Therefore, our finding from a Kurdish region of Iran, although the first of its type in western part of Iran, can be generalized to the whole Iranian population. ${ }^{28,29}$

\section{Conclusion}

Although the prevalence of self-report diabetes is frequently used by different studies, in Iran (similar to other studies) it provide an underestimate of real prevalence and is higly dependent to sociodemographic factors such as age, gender, education and family history of diabetes.

\section{Acknowledgements}

RaNCD is part of the PERSIAN national cohort, and we would like to thank Prof. Reza Malekzadeh, the Deputy of Research and Technology at the Ministry of Health and Medical Education of Iran and Director of the PERSIAN cohort, as well as Dr. Hossein Poustchi, the Executive Director of the PERSIAN cohort, for all their support during the design and implementation of the RaNCD cohort. This study was supported by the Ministry of Health and Medical Education of Iran and Kermanshah University of Medical Sciences (grant No. 92472).

\section{Appendix A. Supplementary data}

Supplementary data to this article can be found online at https:// doi.org/10.1016/j.cegh.2019.04.010.

\section{References}

1. Association AD. Standards of medical care in diabetes-2014. Diabetes Care. 2014;37(Supplement 1):S14-S80.

2. Golozar A, Khademi H, Kamangar F, et al. Diabetes mellitus and its correlates in an Iranian adult population. PLoS One. 2011;6(10) e26725.

3. Mokdad AH. Diabetes mellitus and chronic kidney disease in the eastern mediterranean region: findings from the global burden of disease 2015 study. Int J Public Health. 2017:1-10.

4. Murray CJ, Barber RM, Foreman KJ, et al. Global, regional, and national disabilityadjusted life years (DALYs) for 306 diseases and injuries and healthy life expectancy (HALE) for 188 countries, 1990-2013: quantifying the epidemiological transition. The Lancet. 2015;386(10009):2145-2191.

5. Khorrami Z, Yarahmadi S, Etemad K, Khodakarim S, Kameli ME, Hazaveh ARM Urban-rural differences in the prevalence of self-reported diabetes and its risk factors: the WHO STEPS Iranian noncommunicable disease risk factor surveillance in 2011. Iran J Med Sci. 2017;41(2).

6. Schneider AL, Pankow JS, Heiss G, Selvin E. Validity and reliability of self-reported diabetes in the atherosclerosis risk in communities study. Am. J. Epidemiol. 2012;176(8):738-743.

7. Okura Y, Urban LH, Mahoney DW, Jacobsen SJ, Rodeheffer RJ. Agreement between self-report questionnaires and medical record data was substantial for diabetes, hypertension, myocardial infarction and stroke but not for heart failure. $J$ Clin Epidemiol. 2004;57(10):1096-1103.

8. Goldman N, Lin I-F, Weinstein M, Lin Y-H. Evaluating the quality of self-reports of hypertension and diabetes. J Clin Epidemiol. 2003;56(2):148-154.

9. Huerta JM, Tormo MJ, Egea-Caparrós JM, Ortolá-Devesa JB, Navarro C. Accuracy of self-reported diabetes, hypertension, and hyperlipidemia in the adult Spanish population. DINO study findings. Revista Española de Cardiología (English Edition). 2009;62(2):143-152.

10. El Fakiri F, Bruijnzeels MA, Hoes AW. No evidence for marked ethnic differences in accuracy of self-reported diabetes, hypertension, and hypercholesterolemia. J Clin Epidemiol. 2007;60(12):1271-1279.

11. Merkin SS, Cavanaugh K, Longenecker JC, Fink NE, Levey AS, Powe NR. Agreement of self-reported comorbid conditions with medical and physician reports varied by disease among end-stage renal disease patients. J Clin Epidemiol. 2007;60(6):634-642

12. Molenaar EA, Ameijden EJV, Grobbee DE, Numans ME. Comparison of routine care self-reported and biometrical data on hypertension and diabetes: results of the Utrecht Health Project. Eur J Public Health. 2006;17(2):199-205.

13. Poustchi H, Eghtesad S, Kamangar F, Etemadi A, Keshtkar AA, Hekmatdoost A, et al. 
Prospective epidemiological research studies in Iran (the PERSIAN Cohort Study): rationale, objectives, and design. Am J Epidemiol. 2018;187(4):647-655.

14. Eghtesad S, Mohammadi Z, Shayanrad A, Faramarzi E, Joukar F, Hamzeh B, et al. The PERSIAN cohort: providing the evidence needed for healthcare reform. Arch Iran Med. 2017;20(11):691-695.

15. Pasdar Y, Najafi F, Moradinazar M, Shakiba E, Karim H, HamzehB, et al. Cohort profile: Ravansar Non-Communicable Disease cohort study: the first cohort study in a Kurdish population. J Epidemiol. 2019https://doi.org/10.1093/ije/dyy296.

16. Rutstein SO, Johnson K, MEASURE OM. The DHS Wealth Index ORC Macro. MEASURE DHS; 2004.

17. Tanjani PT, Moradinazar M, Mottlagh ME, Najafi F. The prevalence of diabetes mellitus (DM) type II among Iranian elderly population and its association with other age-related diseases, 2012. Arch Gerontol Geriatr. 2015;60(3):373-379.

18. Fontanelli MdM, Teixeira JA, Sales CH, et al. Validation of self-reported diabetes in a representative sample of São Paulo city. Rev Saude Publica. 2017:51.

19. Wu S-C, Li C, Ke D. The agreement between self-reporting and clinical diagnosis for selected medical conditions among the elderly in Taiwan. Publ Health 2000;114(2):137-142.

20. Chun H, Kim I-H, Min K-D. Accuracy of self-reported hypertension, diabetes, and hypercholesterolemia: analysis of a representative sample of Korean older adults. Osong Public Health Research Perspectives. 2016;7(2):108-115.

21. Barra L, Arsenault-Mehta K, Pope J, Hitchon C, Boire G. Validation of self-reported cardiovascular disease and associated Co-morbidities in a large Canadian cohort of early inflammatory arthritis. Rheumatology. 2017;7(211) 2161-1149.1000211.
22. Simpson CF, Boyd CM, Carlson MC, Griswold ME, Guralnik JM, Fried LP. Agreement between self-report of disease diagnoses and medical record validation in disabled older women: factors that modify agreement. J Am Geriatr Soc. 2004;52(1):123-127.

23. Dalstra JA, Kunst AE, Borrell C, et al. Socioeconomic differences in the prevalence of common chronic diseases: an overview of eight European countries. Int $J$ Epidemiol. 2005;34(2):316-326

24. Soriguer F, Goday A, Bosch-Comas A, et al. Prevalence of diabetes mellitus and impaired glucose regulation in Spain: the Di@ bet. es Study. Diabetologia. 2012;55(1):88-93.

25. Chang SA. Smoking and type 2 diabetes mellitus. Diabetes Metabol. $J$. 2012;36(6):399-403.

26. Metzger M, Goldberg M, Chastang J, Leclerc A, Zins M. Factors associated with selfreporting of chronic health problems in the French GAZEL cohort. $J$ Clin Epidemiol 2002;55(1):48-59.

27. Michimi A, Ellis-Griffith G, Lartey G, Ellis-Griffith C, Hunt M. Variability between self-reported diabetes and measured glucose among health screening participants in South Central Kentucky. Diabetes Prim. Care. 2014;8(1):31-38.

28. Haghdoost A, Rezazadeh Kermani M, Sadghirad B, Baradaran H. Prevalence of Type 2 Diabetes in the Islamic Republic of Iran: Systematic Review and Meta-Analysis. 2009; 2009.

29. Khodaeian M, Enayati S, Tabatabaei-Malazy O, Amoli MM. Association between genetic variants and diabetes mellitus in Iranian populations: a systematic review of observational studies. J. Diabetes Res. 2015;2015. 